\title{
Characterization of poplar GrxS14 in different structural forms
}

\section{Dear Editor,}

Glutaredoxins (Grxs) are glutathione-dependent thiol disulfide oxidoreductases of the thioredoxin family present in all organisms from bacteria to human (Noguera et al., 2005). Depending on their active site sequence, Grxs are essentially classified into three families: the dithiol Grxs, the monothiol Grxs and the CC type restricted to plants (Rouhier et al., 2008). Grxs play important biological functions in plants, such as oxidative stress responses, iron-sulfur (FeS) cluster assembly, and cell signaling, etc. (Rouhier et al., 2008). There are totally $31 \mathrm{Grxs}$ isoforms in Arabidopsis thaliana, and 19 Grx isoforms in Populus trichocarpa (Rouhier et al., 2004). For Grxs of Populus trichocarpa, structures of GrxC1, GrxC4 and GrxS12 have been resolved, all belong to dithiol Grxs (Noguera et al., 2005; Feng et al., 2006; Rouhier et al., 2007; Couturier et al., 2009). The only structure available for monothiol Grxs in plants is that of Arabidopsis Grxcp, which is also called GrxS14 or CAXIP1 (Cheng and Hirschi, 2003; Li et al., 2010).

It was found that Arabidopsis GrxS14 is a new class of signaling molecules in plants that can regulate the $\mathrm{Ca}^{2+}$ transport activity of CAX1 (cation exchangers) by interacting with the N-terminal region of CAX1 (Cheng and Hirschi, 2003). It was suggested that Arabidopsis GrxS14 functions to protecting cells against protein oxidative damage (Cheng et al., 2006). Both Arabidopsis and poplar GrxS14 are monothiol Grxs located in the chloroplasts, which exist as an apo form and a holo form bridged by a [2Fe-2S] cluster with two external glutathione (GSH) ligands, and they can complement a yeast grx5 mutant defective in FeS cluster assembly in vivo (Bandyopadhyay et al., 2008). It was proposed that Arabidopsis and poplar GrxS14 may function as scaffold protein for the assembly of [2Fe-2S] cluster, as GrxS14 can transfer intact cluster to physiologically relevant acceptor proteins which is regulated by GSH (Bandyopadhyay et al., 2008; Wang et al., 2012; Liu et al., 2013).

Here we report the solution structure of reduced poplar GrxS14 and structure models for the non-covalent apo GrxS14 dimer and GrxS14/GSH complex, as well as the NMR characterization of holo GrxS14.

The quality of the 2D ${ }^{1} \mathrm{H}-{ }^{15} \mathrm{~N}$ HSQC spectrum of apo GrxS14 at $1 \mathrm{mmol} / \mathrm{L}$ concentration was very poor (Fig. S1), and very few signals could be observed in 3D triple-resonance NMR spectra. Dilution of the sample did not improve the quality of NMR spectra very significantly. Interestingly, much better NMR spectra were obtained with the addition of GSH (Fig. S1). Although apo GrxS14 appeared to be a monomer on the gel filtration column, analytical ultracentrifugation analysis showed two peaks (with molecular weight about $24 \mathrm{kDa}$ and $12 \mathrm{kDa}$ ) for apo GrxS14 without GSH, while there was only one peak at $\sim 12 \mathrm{kDa}$ for apo GrxS14 with GSH (Fig. S2A and S2B). All these suggest that apo GrxS14 should be in a monomer-to-dimer equilibrium, and the dimerization can be inhibited by GSH. Thus, there exists another type of GrxS14 dimer in addition to the holo GrxS14 dimer assembled with a [2Fe-2S] cluster. Based on the analytical ultracentrifugation data (Fig. S2A), the dissociation constant of GrxS14 monomer-to-dimer equilibrium is estimated to be $\sim 0.4 \mathrm{mmol} / \mathrm{L}$.

We determined the solution structure of reduced monomeric GrxS14 using NMR data collected on protein samples in the presence of $20 \mathrm{mmol} / \mathrm{L} \mathrm{GSH}$. A summary of structural restraints used in the structure calculation and statistics for the structure ensemble is listed in Table S1. Residues 5-109 of apo GrxS14 form a compact thioredoxin fold structure while the first four residues are flexible (Fig. 1A). It comprises five $\alpha$-helices and four $\beta$-strands, the four $\beta$-strands constitute a mixed $\beta$-sheet as the core of structure (Fig. 1B). Helices $\alpha 1$ and $\alpha 3$ are packed on one side of the $\beta$-sheet, while $\alpha 2, \alpha 4$ and $\alpha 5$ are on the other side.

The overall fold of poplar GrxS14 is similar to other Grxs. The RMSD of backbone heavy atoms in secondary structure regions is $1.7 \AA$ between poplar and Arabidopsis GrxS14 which shares an $80 \%$ sequence identity (Figs. 1D and S3A). The relatively large RMSD between the two may be due to that the poplar apo GrxS14 was determined in the presence of GSH, while no GSH is in the crystal structure of Arabidopsis GrxS14. When comparing to dithiol Grxs, the major difference is at the loop region between $\beta 1$ and $\alpha 2$ : poplar GrxS14 contains ten amino acid residues, while only four residues in poplar GrxC1 and human Grx2 (Fig. 1C). Sequence alignment indicated that monothiol Grxs all possess a long loop in this region, whereas the dithiol Grxs usually have a short loop (Fig. S3). This long loop before the active site (CGFS) is a structural characteristic of monothiol Grxs. 
A

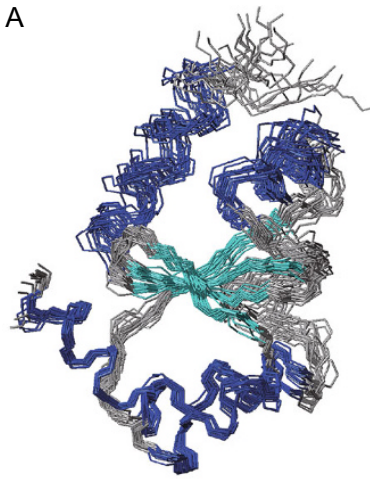

C

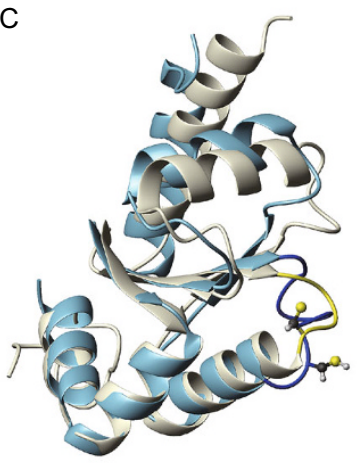

B

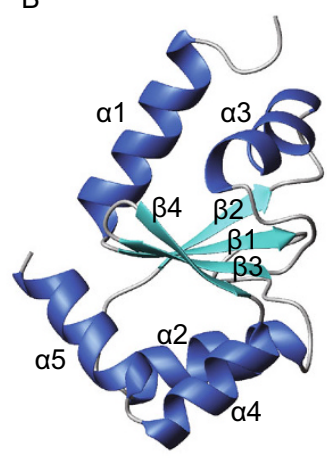

D

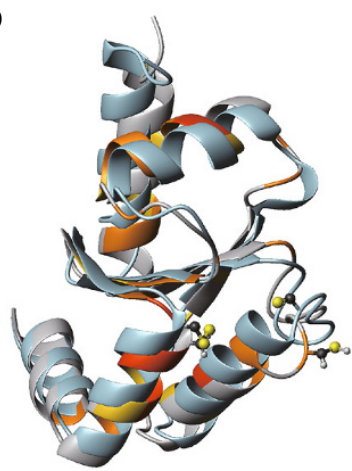

Figure 1. Solution structure and structural comparison of reduced apo GrxS14. (A) The backbone superimposition of 20 structures. (B) The ribbon representation of the mean structure. (C) Structural comparison of poplar GrxS14 (sky blue) and poplar GrxC1 (light yellow) (PDB 1Z7P, 1Z7R). Loop regions between $\beta 1$ and $\alpha 2$ are shown as blue and yellow, respectively. (D) Structural comparison of poplar GrxS14 (light gray) and Arabidopsis GrxS14 (light blue) (PDB 3IPZ). Residues with significant combined $\mathrm{NH}$ chemical shift changes upon $\mathrm{GSH}$ binding are indicated on the structure of poplar GrxS14 by yellow color $(0.05<\delta<0.1 \mathrm{ppm})$, orange color $(0.1 \leq \delta<0.2 \mathrm{ppm})$, and orange red $(\delta \geq 0.2 \mathrm{ppm})$, respectively. The side-chains of conserved active site cysteine are illustrated as ball and stick.

The dimerization of apo GrxS14 was investigated using NMR spectroscopy. Comparison of $2 \mathrm{D}^{1} \mathrm{H}^{-15} \mathrm{~N}$ HSQC spectra of apo GrxS14 at different concentrations revealed that residues with significant concentration-dependent $\mathrm{NH}$ chemical shift changes are F35, Q37, K66, W71, G86, D88, 189, V91, E92 and S96, and these residues should be involved in the dimer interface (Figs. 2A and S4). Based on the structure models of apo GrxS14 dimer calculated using HADDOCK 2.0 (supplementary methods), the dimer interface is located at helices $\alpha 2, \alpha 3$ and $\alpha 4$, and loops $\beta 1-\alpha 2$, $\alpha 3-\beta 3$ and $\beta 4-\alpha 4$ (Fig. 2B). In the dimer interface, there should be aromatic stacking interaction between the two phenyl groups of $\mathrm{F} 35$ since the two rings are close, and sidechains of K66 from one molecule and D88 or E92 from the other are in a distance to form salt bridges ( 2 $\AA$ ) (Fig. 2B).

To verify these interactions, two mutants $\mathrm{F} 35 \mathrm{~A}$ and $\mathrm{D} 88 \mathrm{~A} /$ E92A of GrxS14 were generated. Analytical ultracentrifugation analysis revealed that the amount of dimer fraction is significantly reduced for the two mutants without GSH (Figs. S2C and $\mathrm{S} 2 \mathrm{D}$ ), indicating that the aromatic stacking and the salt bridges are critical for the dimerization. Thus, the docking model of the GrxS14 dimer is valid.

While most Grxs are found to be monomeric protein (Lillig et al., 2008), it was reported that the reduced poplar GrxC4 can self-associate into dimers with $K_{\mathrm{d}}$ in $\mathrm{mmol} / \mathrm{L}$ range (Noguera et al., 2005). Comparing the dimer interface between $\mathrm{GrxS} 14$ and $\mathrm{GrxC} 4$, the residues involved aromatic stacking ( $\mathrm{F} 35$ in GrxS14 and Y29 in GrxC4) and electrostatic interactions (D88, E92 in GrxS14 and D85, E89 in GrxC4) are conserved in sequences (Fig. S3B), and the dimer interface of GrxS14 should be similar to that of GrxC4 (Noguera et al., 2005). Although there is only one molecule in the crystallographic asymmetric unit for the crystal structure of Arabidopsis GrxS14 (Li et al., 2010), we found that a similar dimer interface exists between two molecules in two asymmetric units (Fig. S5B). The dimer interface of Arabidopsis GrxS14 is mainly involved in aromatic contact between two F99 residue and electrostatic interaction between K130 and E156, similar to those in poplar apo GrxS14 dimer (Fig. S5). However, it is expected that there are differences for the details of the two dimer interfaces, since our poplar apo GrxS14 dimer structure is a docking model and the dimer interface for Arabidopsis GrxS14 may be distorted due to crystal packing.

We have also performed NMR titration experiments to monitor the interaction between apo GrxS14 and GSH. The perturbation of GSH on $\mathrm{NH}$ signals of apo GrxS14 is rather dramatic, as residues with significant combined $\mathrm{NH}$ chemical shift changes $(>0.05 \mathrm{ppm})$ between free protein and that with 230 -fold of GSH are distributed on all secondary structure elements (Figs. $2 \mathrm{C}$ and S6). This is consistent with the above mentioned relatively large RMSD between Arabidopsis GrxS14 and poplar apo GrxS14, as our poplar apo GrxS14 structure is determined in the presence of GSH (Fig. 1D). This may suggest that GSH binding can trigger relatively global conformation adjustment for poplar apo GrxS14.

$\mathrm{NH}$ signals from residues at different areas were chosen to fit the dissociation constant and similar $K_{\mathrm{d}}$ values (apparent $K_{\mathrm{d}} \sim 5 \mathrm{mmol} / \mathrm{L}$ ) were obtained, which suggests that only one GSH binds GrxS14 (Fig. S7). Based on the chemical shift perturbation of GSH, we calculated structure models of GSH bound GrxS14 using HADDOCK 2.0 (supplementary methods), which reveal that GSH does bind at the conserved GSH binding motif of Grxs (Lillig et al., 2008). In the models, GSH mainly contacts three regions of GrxS14 (Fig. 2D): loop $\beta 1-\alpha 2$ and $\alpha 3$ (K25 and R62) stabilize the C-terminus of GSH through hydrogen bonds and electrostatic interactions; the GGCD region (between $\beta 4$ and $\alpha 4$ ) stabilizes the $\mathrm{N}$-terminus of $\mathrm{GSH}$ through hydrogen bond and dipolar interaction ( $\alpha 4$ helix); and the WPTFP loop (between $\alpha 3$ and $\beta 3$ ) is well conserved in Grxs, especially 


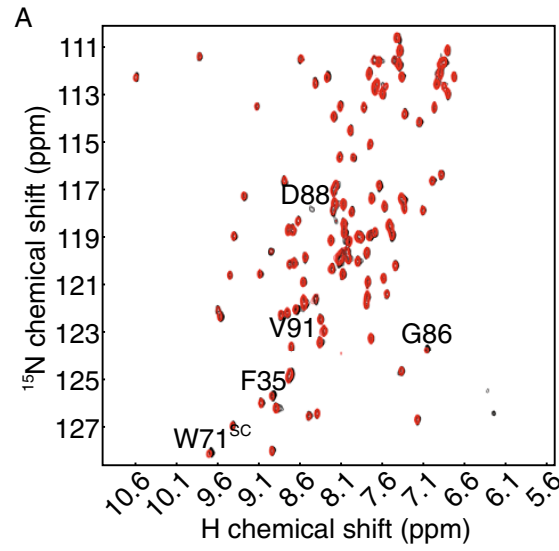

B

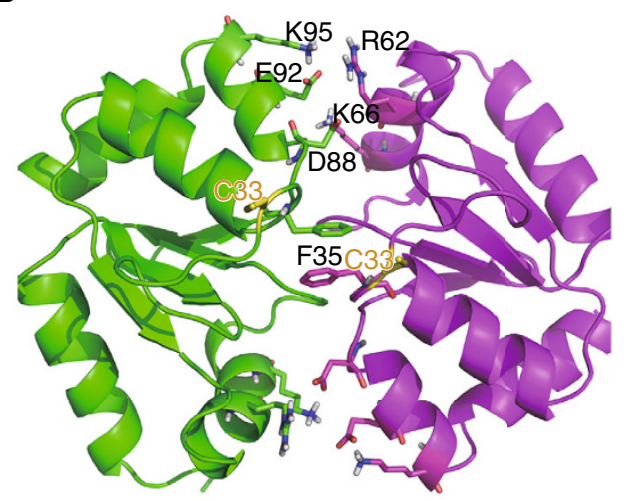

C

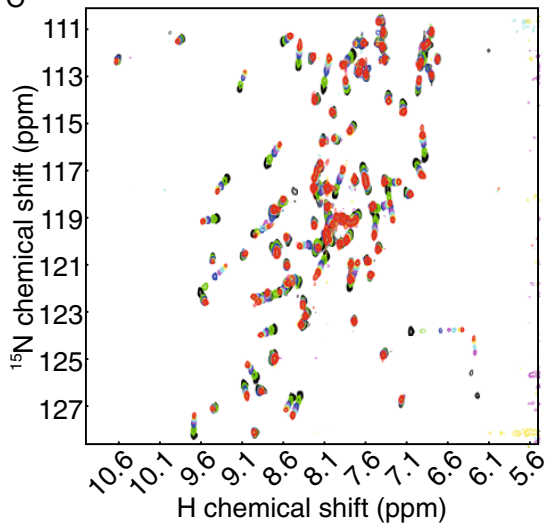

D

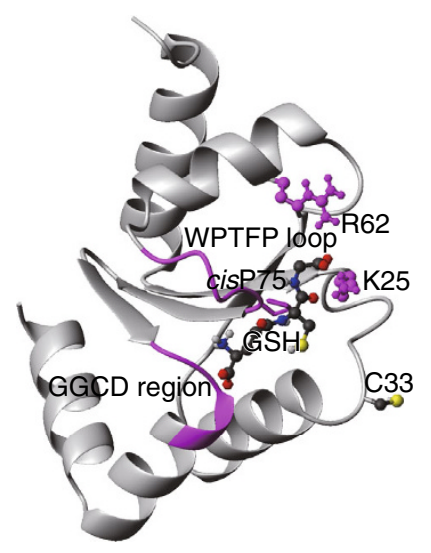

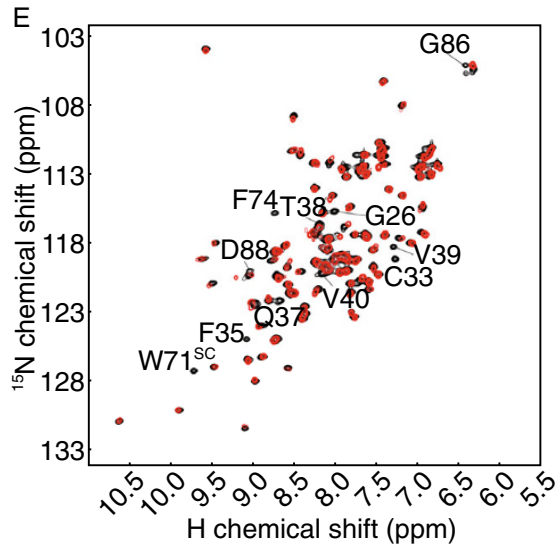

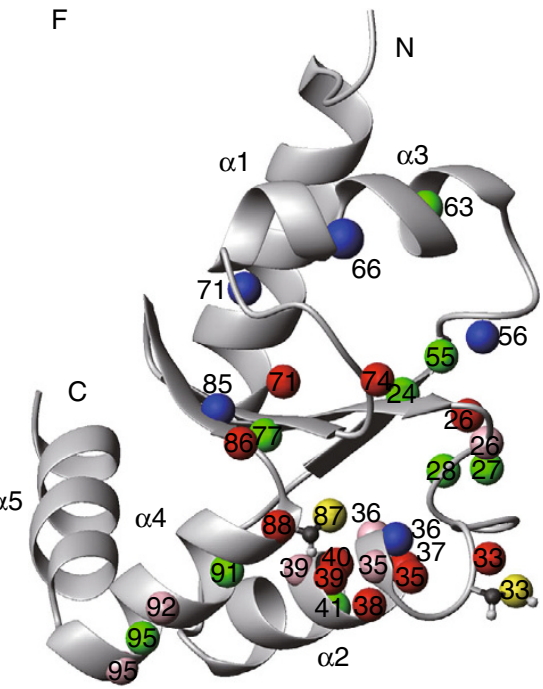

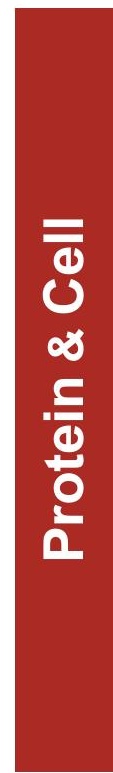

Figure 2. Structural characterization of apo GrxS14 dimer, GrxS14/GSH complex, and holo GrxS14. (A) Superimposed 2D ${ }^{1} \mathrm{H}-{ }^{15} \mathrm{~N} \mathrm{HSQC}$ spectra of apo GrxS14 at $0.1 \mathrm{mmol} / \mathrm{L}$ (black) and $0.4 \mathrm{mmol} / \mathrm{L}$ (red). (B) A structure model of apo GrxS14 dimer (in green and magenta). Side-chains of some interface residues are shown. Side-chains of the active site C33 residues are shown as yellow. (C) $2 \mathrm{D}{ }^{1} \mathrm{H}-{ }^{15} \mathrm{~N} \mathrm{HSQC}$ spectra of GSH titration of apo GrxS14 $(0.1 \mathrm{mmol} / \mathrm{L})$. The colors represent different [GSH]:[GrxS14] ratios from 0 (black) to 230:1 (red). (D) Structure models of GrxS14/GSH complex. GSH is shown as ball and stick. The GSH binding site of GrxS14 is labeled with residues number and colored in magenta. The side-chains of K25 and R62 are also shown in magenta. The active site $\mathrm{C} 33$ is also indicated. (E) Overlay of $2 \mathrm{D}{ }^{1} \mathrm{H}-{ }^{15} \mathrm{~N}$ HSQC spectra of holo (red) and apo (black) poplar GrxS14. Only the assignments of residues with missing $\mathrm{NH}$ signals in holo protein are labeled. ( $F$ ) Mapping of residues with large chemical shift changes or peak intensity changes. Nitrogen atoms corresponding to missing NH peaks in holo GrxS14 are shown as red spheres, those with large intensity reduction are shown as green spheres, and those with large chemical shift changes are shown as blue spheres. The $\mathrm{C}^{\alpha}$ atoms of residues with large chemical shift changes are shown as pink spheres. The sulfur atoms of C33 and C87 are shown as yellow spheres.

the cis configuration of $\mathrm{P} 75$, should provide enhanced favorable contacts with GSH (Couturier et al., 2009). The structural model for GrxS14/GSH complex may provide a recognition mechanism for Grxs to target glutathionylated protein substrates in the reversible protein glutathionylation process. In addition, as the GSH binding site was included in the dimer interface of apo GrxS14 (Figs. 2B, 2D and S8C), the binding of GSH is incompatible with the dimerization of GrxS14, which explains why GSH can prevent the dimerization of GrxS14 (Fig. S2B).
Grx structures with GSH covalently linked to the active site cysteine by disulfide have been reported for $E$. coli $G r \times 3$ (PDB 3GRX), human Grx1 (PDB 1B4Q), yeast Grx1 (PDB 3C1R), Arabidopsis GrxC5 (PDB 3RHB) and poplar GrxS12 (PDB 3FZ9). Grx structures with non-covalently bound $G S H$ are also available for human Grx2 (PDB 2FLS), poplar GrxC4 (Noguera et al., 2005) and yeast Grx6 (PDB 3L4N), all belong to the classical dithiol Grxs. It is found that the GSH binding modes are quite similar at the conserved GSH binding motif (three regions mentioned above) for both monothiol and dithiol Grxs, 
whether GSH bound non-covalently or covalently with mixed disulfide (Figs. S8A and S8B). Meanwhile, ligand GSHs in holo Grxs also show very similar binding modes at the conserved GSH binding motif (Fig. S8B).

Furthermore, we have characterized the holo GrxS14 with NMR spectroscopy. The backbone resonance assignments of holo GrxS14 were determined for $\sim 90 \%$ of the total residues (Wang et al., 2011). A comparison of the $2 \mathrm{D}^{1} \mathrm{H}^{15} \mathrm{~N}$ HSQC spectra of apo and holo GrxS14 is shown in Fig. 2E. NH peaks of residues G26, C33, F35, Q37, T38, V39, V40, W71 (side chain NH), F74, G86 and D88 are missing (Fig. 2E). Most of ${ }^{13} \mathrm{C}^{\alpha}$ chemical shifts are obtained for holo GrxS14, except for residues P31, C33, G34, T73 and G86. On the other hand, only 5 residues have significant $\mathrm{NH}$ chemical shift changes $(\delta>0.05 \mathrm{ppm})$ among the 86 assigned $\mathrm{NH}$ signals: S36, L56, K66, W71, G85 and V91 (Figs. 2F and S9A). NH signals of residues M24, T27, K28, Q41, 155, Q63, L77, V91 and K95 show significantly intensity reduction compared to the apo form (Figs. $2 \mathrm{~F}$ and S9B). The ${ }^{13} \mathrm{C}^{\alpha}$ chemical shift changes are quite small for most residues except for G26, F35, S36, V39, $\mathrm{E} 92$ and $\mathrm{K} 95\left(\delta_{\mathrm{C} \alpha}>0.3 \mathrm{ppm}\right.$ ) (Figs. $2 \mathrm{~F}$ and S9C).

Most of missing and weaker $\mathrm{NH}$ signals should be due to the paramagnetic effect of the [2Fe-2S] cluster, as they are from residues located around the CGFS active site and/or the GSH binding site, where the [2Fe-2S] cluster is presumably coordinated by the active site cysteines and the two GSH cysteines (Fig. 2F). The paramagnetism of the [2Fe-2S] cluster could cause NMR signals broadened and/or hyperfineshifted for residues over $10 \AA$ away from the cluster, dependent on the magnetic susceptibility tensor of the cluster (Feng et al., 2006). Thus, although some of the residues are not very close to the active site, their $\mathrm{NH}$ signals are also affected. Since most $\mathrm{NH}$ peaks in the $2 \mathrm{D}^{1} \mathrm{H}-{ }^{15} \mathrm{~N}$ HSQC spectrum of holo GrxS14 can be superimposed on those of apo GrxS14, and the residues without significant ${ }^{13} \mathrm{C}^{\alpha}$ chemical shift changes are distributed in all secondary structure elements (Fig. 2E and 2F), the structure of each subunit in the holo GrxS14 should largely remain the same as the apo protein.

As a summary, we have determined the solution structure of apo GrxS14, and investigated the structures of the apo GrxS14 dimer, its complex with GSH, and holo GrxS14. The conserved GSH binding site in apo GrxS14 implies the recognition mechanism of monothiol Grxs to various glutathionylated proteins as substrates. While GSH serves as FeS cluster ligand in holo GrxS14, GSH also inhibits the noncovalent dimerization of apo GrxS14. As the $K_{d}$ values of GSH binding is in $\mathrm{mmol} / \mathrm{L}$ level and the concentration of GSH is fluctuating in the $\mathrm{mmol} / \mathrm{L}$ range in plant chloroplast (Rouhier et al., 2008), GSH may have a regulation effect on the dimerization of GrxS14 in vivo. Therefore, it seems that GSH may play a more complicated role in the structure and

Electronic supplementary material The online version of this article (doi:10.1007/s13238-014-0042-3) contains supplementary material, which is available to authorized users. function of GrxS14, as we have previously reported that GSH can regulate the FeS cluster transfer from holo GrxS14 to apo ferredoxin (Wang et al., 2012). Further studies are needed to uncover the hidden physiological roles of GSH on GrxS14.

\section{FOOTNOTES}

All NMR experiments were carried out at the Beijing NMR Center. This research was supported by grants 2011IM030300 and 2006DFA31210 from Ministry of Science and Technology of China, grant CERS-1-51 from Ministry of Education of China, and grant 30570353 from the National Science Foundation of China to BX; and the ANR grant 2010 BLAN 1616 to NR and JPJ.

Lei Wang, Yifei Li, Jean-Pierre Jacquot, Nicolas Rouhier, and Bin Xia declare that they have no conflict of interest. This article does not contain any studies with human or animal subjects performed by any of the authors.

\section{OPEN ACCESS}

This article is distributed under the terms of the Creative Commons Attribution License which permits any use, distribution, and reproduction in any medium, provided the original author(s) and the source are credited.

Lei Wang ${ }^{1,2}$, Yifei $\mathrm{Li}^{1}$, Jean-Pierre Jacquot ${ }^{4}$, Nicolas Rouhier ${ }^{4}$, Bin Xia ${ }^{1,2,3 凶}$

${ }^{1}$ Beijing Nuclear Magnetic Resonance Center, Peking University, Beijing 100871, China

2 College of Chemistry and Molecular Engineering, Peking University, Beijing 100871, China

${ }^{3}$ College of Life Science, Peking University, Beijing 100871, China

${ }^{4}$ Unité Mixte de Recherches INRA UHP 1136, Interaction Arbres Microorganismes, IFR 110 Genomique, Ecophysiologie et Ecologie Fonctionnelles Université Henri Poincaré, BP 239, 54506 Vandoeuvre Cedex, France

$\bowtie$ Correspondence: binxia@pku.edu.cn (B. Xia)

\section{REFERENCES}

Bandyopadhyay S, Gama F, Molina-Navarro MM, Gualberto JM, Claxton R, Naik SG, Huynh BH, Herrero E, Jacquot JP, Johnson MK et al (2008) Chloroplast monothiol glutaredoxins as scaffold proteins for the assembly and delivery of [2Fe-2S] clusters. EMBO J 27:1122-1133

Cheng NH, Hirschi KD (2003) Cloning and characterization of CXIP1, a novel PICOT domain-containing Arabidopsis protein that associates with CAX1. J Biol Chem 278:6503-6509

Cheng NH, Liu JZ, Brock A, Nelson RS, Hirschi KD (2006) AtGRXcp, an Arabidopsis chloroplastic glutaredoxin, is critical for protection against protein oxidative damage. $\mathrm{J}$ Biol Chem 281:26280-26288

Couturier J, Koh CS, Zaffagnini M, Winger AM, Gualberto JM, Corbier C, Decottignies P, Jacquot JP, Lemaire SD, Didierjean C et al (2009) Structure-function relationship of the chloroplastic glutaredoxin S12 with an a typical WCSYS active site. J Biol Chem 284:9299-9310 
Feng Y, Zhong N, Rouhier N, Hase T, Kusunoki M, Jacquot JP, Jin C, Xia B (2006) Structural insight into poplar glutaredoxin C1 with a bridging iron-sulfur cluster at the active site. Biochemistry 45:7998-8008

Li L, Cheng N, Hirschi KD, Wang X (2010) Structure of Arabidopsis chloroplastic monothiol glutaredoxin AtGRXcp. Acta Crystallogr 66:725-732

Lillig CH, Berndt C, Holmgren A (2008) Glutaredoxin systems. Biochim Biophys Acta 1780:1304-1317

Liu X, Liu S, Feng Y, Liu JZ, Chen Y, Pham K, Deng H, Hirschi KD, Wang $X$, Cheng N (2013) Structural insights into the N-terminal GIY-YIG endonuclease activity of Arabidopsis glutaredoxin AtGRXS16 in chloroplasts. Proc Natl Acad Sci USA 110: 9565-9570

Noguera V, Walker O, Rouhier N, Jacquot JP, Krimm I, Lancelin JM (2005) NMR reveals a novel glutaredoxin-glutaredoxin interaction interface. J Mol Biol 353:629-641
Rouhier N, Gelhaye E, Jacquot JP (2004) Plant glutaredoxins: still mysterious reducing systems. Cell Mol Life Sci 61:1266-1277

Rouhier N, Unno H, Bandyopadhyay S, Masip L, Kim SK, Hirasawa M, Gualberto JM, Lattard V, Kusunoki M, Knaff DB et al (2007) Functional, structural, and spectroscopic characterization of a glutathione-ligated [2Fe-2S] cluster in poplar glutaredoxin $\mathrm{C} 1$. Proc Natl Acad Sci USA 104:7379-7384

Rouhier N, Lemaire SD, Jacquot JP (2008) The role of glutathione in photosynthetic organisms: emerging functions for glutaredoxins and glutathionylation. Annu Rev Plant Biol 59:143-166

Wang L, Ren X, Li Y, Rouhier N, Jacquot JP, Jin C, Xia B (2011) ${ }^{1} \mathrm{H}$, ${ }^{13} \mathrm{C}$, and ${ }^{15} \mathrm{~N}$ resonance assignments of reduced $\mathrm{GrXS} 14$ from Populus tremula $\times$ tremuloides. Biomol NMR Assign 5:121-124

Wang L, Ouyang B, Li Y, Feng Y, Jacquot JP, Rouhier N, Xia B (2012) Glutathione regulates the transfer of iron-sulfur cluster from monothiol and dithiol glutaredoxins to apo ferredoxin. Protein Cell 3:714-721 